\title{
Three-Valued Paraconsistent Reasoning for Semantic Web Agents ${ }^{\star}$
}

\author{
Linh Anh Nguyen ${ }^{1}$ and Andrzej Szałas ${ }^{1,2}$ \\ 1 Institute of Informatics, University of Warsaw \\ Banacha 2, 02-097 Warsaw, Poland \\ \{nguyen, andsz\}@mimuw. edu.pl \\ 2 Dept. of Computer and Information Science, Linköping University \\ SE-581 83 Linköping, Sweden
}

\begin{abstract}
Description logics [1] refer to a family of formalisms concentrated around concepts, roles and individuals. They are used in many multiagent and semantic web applications as a foundation for specifying knowledge bases and reasoning about them. One of widely applied description logics is $\mathcal{S H \mathcal { I }}[\mathbf{7} \mid 8]$. In the current paper we address the problem of inconsistent knowledge. Inconsistencies may naturally appear in the considered application domains, for example as a result of fusing knowledge from distributed sources. We define three threevalued paraconsistent semantics for $\mathcal{S H \mathcal { L }}$, reflecting different meanings of concept inclusion of practical importance. We also provide a quite general syntactic condition of safeness guaranteeing satisfiability of a knowledge base w.r.t. threevalued semantics and define a faithful translation of our formalism into a suitable version of a two-valued description logic. Such a translation allows one to use existing tools and $\mathcal{S H I Q}$ reasoners to deal with inconsistent knowledge.
\end{abstract}

\section{Introduction}

Description logics [1] refer to a family of formalisms concentrated around concepts, roles and individuals. They belong to the most frequently used knowledge representation formalisms and provide a logical basis to a variety of well known paradigms, including frame-based systems, semantic networks and semantic web ontologies and reasoners. In particular, description logics are the foundations of the Web Ontology Language OWL [7], as the main goal of description logics is to specify concepts and concept hierarchies and to reason about them.

Description logics have usually been considered as syntactic variants of restricted versions of classical first-order logic. On the other hand, in semantic web and multiagent applications, knowledge/ontology fusion frequently leads to inconsistencies. A way to deal with inconsistencies is to follow the area of paraconsistent reasoning. There is a rich literature on paraconsistent logics (see, e.g., [4] and references there). A number of researchers have extended description logics with paraconsistent semantics and paraconsistent reasoning methods [13|15]14|11|10|17]. The work [14] studies a constructive version of the basic description logic $\mathcal{A L C}$, but it is not clear how to extend the semantics provided in this work to other description logics. The remaining works are based

\footnotetext{
* Partially supported by grants N N206 399334 and N N206 370739 from the Polish Ministry of Science and Higher Education.
} 
on the well-known Belnap's four-valued logic [3|2]. Truth values in this logic represent truth $(\mathfrak{t})$, falsity $(\mathfrak{f})$, the lack of knowledge $(\mathfrak{u})$ and inconsistency $(\mathfrak{i})$. However, there are serious problems with using Belnap's logic for the semantic web. Some of these problems are considered in the general context, e.g., in [12[16]. We give here some others, more directly related to description logics (see also Section 5):

- According to the semantics given in [13|15|11|10|17], if $\left(x \in C^{\mathcal{I}}\right)=\mathbf{i}$ and $(x \in$ $\left.D^{\mathcal{I}}\right)=\mathfrak{u}$ then $\left(x \in(C \sqcap D)^{\mathcal{I}}\right)=\mathfrak{f}$, which, in our opinion, is not intuitive.

- A knowledge base, as a theory, may be incomplete in the sense that truth value of a formula given as a query to the knowledge base may be not determinable using the theory. In such cases, we have a meta-unknown value. If the semantics uses a truth value $\mathfrak{u}$, one can raise the question about the relationship between $\mathfrak{u}$ and the meta-unknown value. This problem was not addressed in the mentioned works.

- One of the most common approaches is to use paraconsistent reasoning for knowledge bases specified in the traditional way without explicit truth values $\mathfrak{t}, \mathfrak{f}, \mathfrak{i}, \mathfrak{u}$. The reason is that, if we allow explicit uses of $\mathfrak{t}, \mathfrak{f}, \mathfrak{i}, \mathfrak{u}$ then, for example, two facts $C(a): \mathfrak{t}$ and $C(a): \mathfrak{u}$ in a knowledge base form a clash. With this approach, which is used in [13]15]11|10|17], $\mathfrak{u}$ is not used for knowledge representation but only for the semantics, but allowing the value $\mathfrak{u}$ by excluding the axioms $T \sqsubseteq A \sqcup \neg A$ in many cases weakens the logic too much.

In the current paper we model inconsistency using only three truth values $(\mathfrak{t}, \mathfrak{f}, \mathfrak{i})$ as in Kleene's three-valued logic [95]. In a sense, we identify inconsistency with the lack of knowledge. There are many good reasons for such an identification (see, e.g., [6]). Assuming that the objective reality is consistent, the value i reflects a sort of lack of knowledge. Namely, inconsistent information often reflects differences in subjective realities of agents resulting, for example, from their different perceptual capabilities. Inconsistency appears, when different information sources do not agree with one another and one cannot decide which of them is right. Also, in many multiagent and semantic web scenarios one has contradictory evidence as to a given fact. Last, but not least, using three-valued setting, we substantially simplify the underlying formalism.

The main contributions of the current paper are:

- We define three three-valued paraconsistent semantics for $\mathcal{S H I} \mathcal{I}$, reflecting different meanings of concept inclusion $\sqsubseteq$ of practical importance. As discussed in Section 5, our semantics are stronger or more appropriate than the corresponding four-valued semantics defined in [13|15|11|10|17].

- We provide a quite general syntactic condition of safeness guaranteeing satisfiability of a knowledge base w.r.t. three-valued semantics.

- We define a faithful translation of our formalism into a suitable version of a twovalued description logic, showing how to adapt existing tools and reasoners to work with the extended semantics.

The rest of the paper is structured as follows. In Section 2 we recall the two-valued description logic $\mathcal{S H I} \mathcal{I}$. Section 3 provides our three-valued paraconsistent semantics for $\mathcal{S H} \mathcal{I} \mathcal{Q}$. In Section 4 we investigate properties of the new semantics. In Section 5 we discuss the relationship between our three-valued paraconsistent semantics and the four-valued paraconsistent semantics studied in [11[10[17]. Section 6 is devoted to a translation of three-valued $\mathcal{S H \mathcal { I } Q}$ into its standard two-valued version. 


\section{The Two-Valued $\mathcal{S H} \mathcal{H} \mathcal{Q}$}

In this section we recall notation and semantics of the description logic $\mathcal{S H I Q}$ (see, e.g., [7]8]). Assume that our language uses a finite set RNAMES of role names, a finite set CNAMES of concept names, and a finite set INAMES of individual names. Roles are interpreted as binary relations and concepts as unary relations.

Let RNAMES $\stackrel{- \text { def }}{=}\left\{r^{-} \mid r \in\right.$ RNAMES $\}$ be the set of inverse roles. A $(\mathcal{S H I} \mathcal{Q})$ role is any member of RNAMES $\cup$ RNAMES $^{-}$. We use letters like $R$ and $S$ for roles. For any role $R$, we define its inverse $R^{-} \stackrel{\text { def }}{=} r^{-}$if $R=r$, and $R^{-} \stackrel{\text { def }}{=} r$ if $R=r^{-}$.

A $(\mathcal{S H I} \mathcal{I})$ RBox $\mathcal{R}$ is a finite set of role axioms of the form $R \sqsubseteq S$ or $R \circ R \sqsubseteq$ $R$, where $\circ$ stands for the composition of relations. By $\operatorname{ext}(\mathcal{R})$ we denote the least extension of $\mathcal{R}$ such that:

- if $(R \sqsubseteq S) \in \mathcal{R}$ then $\left(R^{-} \sqsubseteq S^{-}\right) \in \operatorname{ext}(\mathcal{R})$

- if $(R \circ R \sqsubseteq R) \in \mathcal{R}$ then $\left(R^{-} \circ R^{-} \sqsubseteq R^{-}\right) \in \operatorname{ext}(\mathcal{R})$

- $(R \sqsubseteq R) \in \operatorname{ext}(\mathcal{R})$, for all $R \in$ RNAMES

- if $(\bar{R} \sqsubseteq S) \in \operatorname{ext}(\mathcal{R})$ and $(S \sqsubseteq T) \in \operatorname{ext}(\mathcal{R})$ then $(R \sqsubseteq T) \in \operatorname{ext}(\mathcal{R})$.

By $R \sqsubseteq \mathcal{R} S$ we mean $(R \sqsubseteq S) \in \operatorname{ext}(R)$. If $R \sqsubseteq \mathcal{R} S$ then $R$ is a sub-role of $S$ w.r.t. $\mathcal{R}$. If $(R \circ R \sqsubseteq R) \in \operatorname{ext}(\mathcal{R})$ then $R$ is a transitive role w.r.t. $\mathcal{R}$.

We call elements of CNAMES atomic concepts and use letters like $A$ and $B$ to denote them. Concepts are denoted by letters like $C, D$ and in $\mathcal{S H \mathcal { Q }}$ they are formed using the following BNF grammar, where $n$ denotes a natural number:

$$
C, D::=\top|\perp| A|\neg C| C \sqcap D|C \sqcup D| \forall R . C|\exists R . C| \geq n R . C \mid \leq n R . C
$$

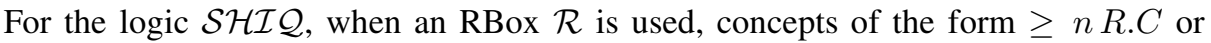
$\leq n R . C$ are allowed only when $R$ is a simple role w.r.t. $\mathcal{R}$, that is, only when $R$ is not a transitive role and does not have any transitive sub-role w.r.t. $\mathcal{R}$.

We use small letters like $a$ and $b$ to denote individuals, i.e. elements of INAMES.

A TBox is a finite set of terminological axioms of the form $C \sqsubseteq D$ or $C=D$. An $A B o x$ is a finite set of assertions of the form $C(a)$ (concept assertion) or $R(a, b)$ (role assertion). A knowledge base is a triple $\langle\mathcal{R}, \mathcal{T}, \mathcal{A}\rangle$, where $\mathcal{R}$ is an RBox, $\mathcal{T}$ is a TBox and $\mathcal{A}$ is an ABox.

A (two-valued) interpretation $\mathcal{I}=\left\langle\Delta^{\mathcal{I}},{ }^{\mathcal{I}}\right\rangle$ consists of a non-empty set $\Delta^{\mathcal{I}}$, called the domain of $\mathcal{I}$, and a function ${ }^{\mathcal{I}}$, called the interpretation function of $\mathcal{I}$, that maps every concept name $A$ to a subset $A^{\mathcal{I}}$ of $\Delta^{\mathcal{I}}$, maps every role name $R$ to a binary relation $R^{\mathcal{I}}$ on $\Delta^{\mathcal{I}}$, and maps every individual name $a$ to an element $a^{\mathcal{I}} \in \Delta^{\mathcal{I}}$. The interpretation function is extended to inverse roles and complex concepts as follows:

$$
\begin{aligned}
& \top^{\mathcal{I}} \stackrel{\text { def }}{=} \Delta^{\mathcal{I}} \quad \stackrel{\perp}{\perp} \stackrel{\text { def }}{=} \emptyset \quad\left(R^{-}\right)^{\mathcal{I}} \stackrel{\text { def }}{=}\left(R^{\mathcal{I}}\right)^{-1} \quad(\neg C)^{\mathcal{I}} \stackrel{\text { def }}{=} \Delta^{\mathcal{I}} \backslash C^{\mathcal{I}} \\
& (C \sqcap D)^{\mathcal{I}} \stackrel{\text { def }}{=} C^{\mathcal{I}} \cap D^{\mathcal{I}} \quad(C \sqcup D)^{\mathcal{I}} \stackrel{\text { def }}{=} C^{\mathcal{I}} \cup D^{\mathcal{I}} \\
& (\forall R . C)^{\mathcal{I}} \stackrel{\text { def }}{=}\left\{x \in \Delta^{\mathcal{I}} \mid \forall y\left[(x, y) \in R^{\mathcal{I}} \text { implies } y \in C^{\mathcal{I}}\right]\right\} \\
& (\exists R . C)^{\mathcal{I}} \stackrel{\text { def }}{=}\left\{x \in \Delta^{\mathcal{I}} \mid \exists y\left[(x, y) \in R^{\mathcal{I}} \text { and } y \in C^{\mathcal{I}}\right]\right\} \\
& (\geq n R . C)^{\mathcal{I}} \stackrel{\text { def }}{=}\left\{x \in \Delta^{\mathcal{I}} \mid \#\left\{y \mid(x, y) \in R^{\mathcal{I}} \text { and } y \in C^{\mathcal{I}}\right\} \geq n\right\} \\
& (\leq n R . C)^{\mathcal{I}} \stackrel{\text { def }}{=}\left\{x \in \Delta^{\mathcal{I}} \mid \#\left\{y \mid(x, y) \in R^{\mathcal{I}} \text { and } y \in C^{\mathcal{I}}\right\} \leq n\right\},
\end{aligned}
$$


where $\# \Gamma$ stands for the number of elements in the set $\Gamma$. We also have the following definitions:

- An interpretation $\mathcal{I}$ satisfies a concept $C$ if $C^{\mathcal{I}} \neq \emptyset$, and validates a concept $C$ if $C^{\mathcal{I}}=\Delta^{\mathcal{I}}$. I satisfies an assertion $C(a)$, denoted by $\mathcal{I}=C(a)$, if $a^{\mathcal{I}} \in C^{\mathcal{I}}$.

- An interpretation $\mathcal{I}$ is a model of an RBox $\mathcal{R}$ if for every axiom $R \sqsubseteq S$ (resp. $R \circ R \sqsubseteq R$ ) of $\mathcal{R}$, we have that $R^{\mathcal{I}} \subseteq S^{\mathcal{I}}$ (resp. $R^{\mathcal{I}} \circ R^{\mathcal{I}} \subseteq R^{\mathcal{I}}$ ). Note that if $\mathcal{I}$ is a model of $\mathcal{R}$ then it is also a model of $\operatorname{ext}(\mathcal{R})$.

- An interpretation $\mathcal{I}$ is a model of a TBox $\mathcal{T}$ if for every axiom $C \sqsubseteq D$ (resp. $C=D$ ) of $\mathcal{T}$, we have that $C^{\mathcal{I}} \subseteq D^{\mathcal{I}}$ (resp. $C^{\mathcal{I}}=D^{\mathcal{I}}$ ).

- An interpretation $\mathcal{I}$ is a model of an $A B o x \mathcal{A}$ if for every assertion $C(a)$ (resp. $R(a, b))$ of $\mathcal{A}$, we have that $a^{\mathcal{I}} \in C^{\mathcal{I}}$ (resp. $\left.\left(a^{\mathcal{I}}, b^{\mathcal{I}}\right) \in R^{\mathcal{I}}\right)$.

- An interpretation $\mathcal{I}$ is a (two-valued) model of a knowledge base $\langle\mathcal{R}, \mathcal{T}, \mathcal{A}\rangle$, denoted by $\mathcal{I} \models\langle\mathcal{R}, \mathcal{T}, \mathcal{A}\rangle$, if it is a model of all $\mathcal{R}, \mathcal{T}$ and $\mathcal{A}$.

- A knowledge base $\langle\mathcal{R}, \mathcal{T}, \mathcal{A}\rangle$ is satisfiable (w.r.t. two-valued semantics) if it has a two-valued model.

- An individual $a$ is an instance of $C$ w.r.t. a knowledge base $\langle\mathcal{R}, \mathcal{T}, \mathcal{A}\rangle$ and twovalued semantics, denoted by $\langle\mathcal{R}, \mathcal{T}, \mathcal{A}\rangle \models C(a)$, if for every two-valued interpretation $\mathcal{I}$, if $\mathcal{I}=\langle\mathcal{R}, \mathcal{T}, \mathcal{A}\rangle$ then $\mathcal{I}=C(a)$.

\section{Three-Valued Paraconsistent Semantics for $\mathcal{S H I \mathcal { Q }}$}

Following the recommendation of W3C for OWL, we use the traditional syntax of description logics and only change its semantics to cover paraconsistency.

Using two-valued semantics and the description logic $\mathcal{S H I} \mathcal{\mathcal { Q }}$, only terminological axioms and concept assertions may directly cause a knowledge base inconsistent, while role axioms and role assertions may not. For this reason, similarly as in [10[11], our paraconsistent semantics for $\mathcal{S H} \mathcal{I} \mathcal{Q}$ employs three-valued interpretations $\mathcal{I}$ which assign value $\mathfrak{t}$ (true), $\mathfrak{f}$ (false) or $\mathfrak{i}$ (inconsistent) to a fact of the form $x \in C^{\mathcal{I}}$, but only assign value $\mathfrak{t}$ or $\mathfrak{f}$ to a fact of the form $\left.(x, y) \in R^{\mathcal{I}}\right]$

A three-valued interpretation $\mathcal{I}=\left\langle\Delta^{\mathcal{I}},{ }^{\mathcal{I}}\right\rangle$ is similar to a two-valued interpretation except that the interpretation function maps every concept name $A$ to a pair $\left\langle A_{+}^{\mathcal{I}}, A_{-}^{\mathcal{I}}\right\rangle$ of subsets of $\Delta^{\mathcal{I}}$ such that $A_{+}^{\mathcal{I}} \cup A_{-}^{\mathcal{I}}=\Delta^{\mathcal{I}}$. The intuition behind $\left\langle A_{+}^{\mathcal{I}}, A_{-}^{\mathcal{I}}\right\rangle$ is that $A_{+}^{\mathcal{I}}$ gathers positive evidence about $A$, while $A_{-}^{\mathcal{I}}$ gathers negative evidence about $A$. Inconsistency appears when a domain object belongs both to $A_{+}^{\mathcal{I}}$ and $A_{-}^{\mathcal{I}}$.

We still define $\left(R^{-}\right)^{\mathcal{I}}=\left(R^{\mathcal{I}}\right)^{-1}$. For a complex concept $C$, the interpretation function maps $C$ to a pair $C^{\mathcal{I}}=\left\langle C_{+}^{\mathcal{I}}, C_{-}^{\mathcal{I}}\right\rangle$ of subsets of $\Delta^{\mathcal{I}}$ defined as follows:

$$
\begin{array}{ll}
\top^{\mathcal{I}} \stackrel{\text { def }}{=}\left\langle\Delta^{\mathcal{I}}, \emptyset\right\rangle \quad \perp \stackrel{\mathcal{I}}{\stackrel{\text { def }}{=}}\left\langle\emptyset, \Delta^{\mathcal{I}}\right\rangle & (\neg C)^{\mathcal{I}} \stackrel{\text { def }}{=}\left\langle C_{-}^{\mathcal{I}}, C_{+}^{\mathcal{I}}\right\rangle \\
(C \sqcap D)^{\mathcal{I}} \stackrel{\text { def }}{=}\left\langle C_{+}^{\mathcal{I}} \cap D_{+}^{\mathcal{I}}, C_{-}^{\mathcal{I}} \cup D_{-}^{\mathcal{I}}\right\rangle & (C \sqcup D)^{\mathcal{I}} \stackrel{\text { def }}{=}\left\langle C_{+}^{\mathcal{I}} \cup D_{+}^{\mathcal{I}}, C_{-}^{\mathcal{I}} \cap D_{-}^{\mathcal{I}}\right\rangle
\end{array}
$$

\footnotetext{
${ }^{1}$ The new version OWL 2 recommended by W3C in October 2009 uses a more expressive

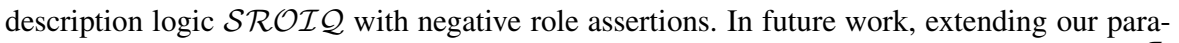
consistent semantics to $\mathcal{S} \mathcal{R O} \mathcal{I} \mathcal{Q}$ we will allow also value $\mathfrak{i}$ for facts of the form $(x, y) \in R^{\mathcal{I}}$.
} 


$$
\begin{aligned}
& (\forall R . C)^{\mathcal{I}} \stackrel{\text { def }}{=}\left\langle\left\{x \in \Delta^{\mathcal{I}} \mid \forall y\left((x, y) \in R^{\mathcal{I}} \text { implies } y \in C_{+}^{\mathcal{I}}\right)\right\},\right. \\
& \left.\left\{x \in \Delta^{\mathcal{I}} \mid \exists y\left((x, y) \in R^{\mathcal{I}} \text { and } y \in C_{-}^{\mathcal{I}}\right)\right\}\right\rangle \\
& (\exists R . C)^{\mathcal{I}} \quad \stackrel{\text { def }}{=}\left\langle\left\{x \in \Delta^{\mathcal{I}} \mid \exists y\left((x, y) \in R^{\mathcal{I}} \text { and } y \in C_{+}^{\mathcal{I}}\right)\right\}\right. \text {, } \\
& \left.\left\{x \in \Delta^{\mathcal{I}} \mid \forall y\left((x, y) \in R^{\mathcal{I}} \text { implies } y \in C_{-}^{\mathcal{I}}\right)\right\}\right\rangle \\
& (\geq n R . C)^{\mathcal{I}} \stackrel{\text { def }}{=}\left\langle\left\{x \in \Delta^{\mathcal{I}} \mid \#\left\{y \mid(x, y) \in R^{\mathcal{I}} \text { and } y \in C_{+}^{\mathcal{I}}\right\} \geq n\right\},\right. \\
& \left.\left\{x \in \Delta^{\mathcal{I}} \mid \#\left\{y \mid(x, y) \in R^{\mathcal{I}} \text { and } y \notin C_{-}^{\mathcal{I}}\right\}<n\right\}\right\rangle \\
& (\leq n R . C)^{\mathcal{I}} \stackrel{\text { def }}{=}\left\langle\left\{x \in \Delta^{\mathcal{I}} \mid \#\left\{y \mid(x, y) \in R^{\mathcal{I}} \text { and } y \notin C_{-}^{\mathcal{I}}\right\} \leq n\right\}\right. \text {, } \\
& \left.\left\{x \in \Delta^{\mathcal{I}} \mid \#\left\{y \mid(x, y) \in R^{\mathcal{I}} \text { and } y \in C_{+}^{\mathcal{I}}\right\}>n\right\}\right\rangle \text {. }
\end{aligned}
$$

Note that $C^{\mathcal{I}}$ of every concept $C$ is computed in the standard way as in [10 11]17]. Hence, properties like De Morgan's laws hold for our constructors. The essential point of our semantics, however, is that we use only three-valued interpretations and have the following property, which can be proved by induction on the structure of $C$.

Proposition 3.1. For every three-valued interpretation $\mathcal{I}$ and every concept $C$, we have that $C_{+}^{\mathcal{I}} \cup C_{-}^{\mathcal{I}}=\Delta^{\mathcal{I}}$.

For a concept $C$ and a three-valued interpretation $\mathcal{I}, C^{\mathcal{I}}$ can be treated as the function from $\Delta^{\mathcal{I}}$ to $\{\mathbf{t}, \mathfrak{f}, \mathfrak{i}\}$ defined below. The value of $C(a)$ in $\mathcal{I}$ is defined to be $C^{\mathcal{I}}\left(a^{\mathcal{I}}\right)$.

$$
C^{\mathcal{I}}(x) \stackrel{\text { def }}{=}\left\{\begin{array}{l}
\mathfrak{t} \text { for } x \in C_{ \pm}^{\mathcal{I}} \text { and } x \notin C^{\mathcal{I}} \\
\mathfrak{f} \text { for } x \in C_{-}^{\mathcal{I}} \text { and } x \notin C_{+}^{\overline{\mathcal{I}}} \\
\mathfrak{i} \text { for } x \in C_{+}^{\mathcal{I}} \text { and } x \in C_{-}^{\overline{\mathcal{I}}}
\end{array}\right.
$$

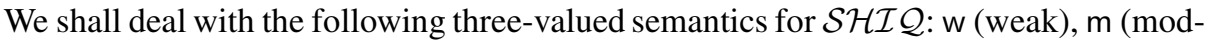
erate) and s (strong). They differ from each other only in interpreting terminological axioms. For a three-valued interpretation $\mathcal{I}$ and $t \in\{\mathrm{w}, \mathrm{m}, \mathrm{s}\}$, we say that:

- I w-validates $C \sqsubseteq D$, denoted by $\mathcal{I} \models_{3}^{\mathrm{w}} C \sqsubseteq D$, if $C_{-}^{\mathcal{I}} \cup D_{+}^{\mathcal{I}}=\Delta^{\mathcal{I}}$

- $\mathcal{I}$ m-validates $C \sqsubseteq D$, denoted by $\mathcal{I}=_{3}^{\mathrm{m}} C \sqsubseteq D$, if $C_{+}^{\mathcal{I}} \subseteq D_{+}^{\mathcal{I}}$

- I s-validates $C \sqsubseteq D$, denoted by $\mathcal{I} \models_{3}^{\mathrm{s}} C \sqsubseteq D$, if $C_{+}^{\mathcal{I}} \subseteq D_{+}^{\mathcal{I}}$ and $D_{-}^{\mathcal{I}} \subseteq C_{-}^{\mathcal{I}}$

- I $t$-validates $C=D$ if it $t$-validates $C \sqsubseteq D$ and $D \sqsubseteq C$

- $\mathcal{I}$ is a $t$-model of a TBox $\mathcal{T}$ if it $t$-validates all axioms of $\mathcal{T}$.

The notion of a model of an RBox remains unchanged. We have the following definitions, where $t \in\{\mathrm{w}, \mathrm{m}, \mathrm{s}\}$.

- A three-valued interpretation $\mathcal{I}$ satisfies an assertion $C(a)$, denoted by $\mathcal{I}=_{3} C(a)$, if $a^{\mathcal{I}} \in C_{+}^{\mathcal{I}}$.

- A three-valued interpretation $\mathcal{I}$ is a model of an $A B o x \mathcal{A}$ if for every assertion $C(a)$ (resp. $R(a, b)$ ) of $\mathcal{A}$, we have that $a^{\mathcal{I}} \in C_{+}^{\mathcal{I}}$ (resp. $\left.\left(a^{\mathcal{I}}, b^{\mathcal{I}}\right) \in R^{\mathcal{I}}\right)$.

- A three-valued interpretation $\mathcal{I}$ is a $t$-model of a knowledge base $\langle\mathcal{R}, \mathcal{T}, \mathcal{A}\rangle$, if it is a model of $\mathcal{R}$ and $\mathcal{A}$ and is a $t$-model of $\mathcal{T}$. In that case we write $\mathcal{I} \models{ }_{3}^{t}\langle\mathcal{R}, \mathcal{T}, \mathcal{A}\rangle$.

- A knowledge base $\langle\mathcal{R}, \mathcal{T}, \mathcal{A}\rangle$ is $t$-satisfiable, if it has a $t$-model.

- We say that $a$ is an instance of $C$ w.r.t. a knowledge base $\langle\mathcal{R}, \mathcal{T}, \mathcal{A}\rangle$ and semantics $t$, denoted by $\langle\mathcal{R}, \mathcal{T}, \mathcal{A}\rangle \models_{3}^{t} C(a)$, if for every three-valued interpretation $\mathcal{I}$, if $\mathcal{I} \models_{3}^{t}\langle\mathcal{R}, \mathcal{T}, \mathcal{A}\rangle$ then $\mathcal{I} \models_{3} C(a)$. 
Example 3.2. Consider a web service supplying information about stocks. Assume that a web agent looks for low risk stocks, promising big gain. The agent's query can be expressed by $(L R \sqcap B G)(x)$, where $L R$ and $B G$ stand for "low risk" and "big gain", respectively. For simplicity, assume that the service has a knowledge base consisting only of the following concept assertions (provided by different experts/agents): $L R\left(s_{1}\right)$, $\neg L R\left(s_{1}\right), B G\left(s_{1}\right), \neg L R\left(s_{2}\right), \neg B G\left(s_{2}\right), L R\left(s_{3}\right), B G\left(s_{3}\right)$. The query looks for stocks $x$ that are instances of $L R \sqcap B G$ w.r.t. the knowledge base. Using two-valued semantics, the knowledge base has no models, and hence all of $s_{1}, s_{2}, s_{3}$ are answers to the query, despite that $s_{2}$ is of high risk and low gain. Using three-valued semantics, only $s_{1}$ and $s_{3}$ are answers to the query, which is well-justified. Namely, there is only positive evidence that $s_{3}$ satisfies the query and there is some positive 2 evidence that $s_{1}$ satisfies the query (resulting from the facts $L R\left(s_{1}\right)$ and $B G\left(s_{1}\right)$ ).

\section{Properties of the Three-Valued Semantics}

The following proposition and corollary state that semantics $s$ is stronger than $\mathrm{m}$, which in turn is stronger than w.

Proposition 4.1. Let $\mathcal{I}$ be a three-valued interpretation. Then:

- if $\mathcal{I} \models_{3}^{\mathrm{s}} C \sqsubseteq D$ then $\mathcal{I} \models_{3}^{\mathrm{m}} C \sqsubseteq D \quad-$ if $\mathcal{I} \models_{3}^{\mathrm{m}} C \sqsubseteq D$ then $\mathcal{I} \models_{3}^{\mathrm{w}} C \sqsubseteq D$

- if $\mathcal{I} \models{ }_{3}^{\mathrm{s}} C=D$ then $\mathcal{I}={ }_{3}^{\mathrm{m}} C=D \quad-$ if $\mathcal{I} \models_{3}^{\mathrm{m}} C=D$ then $\mathcal{I} \models{ }_{3}^{\mathrm{w}} C=D$.

Proof. Just note that $C_{+}^{\mathcal{I}} \subseteq D_{+}^{\mathcal{I}}$ implies $\Delta^{\mathcal{I}}=C_{-}^{\mathcal{I}} \cup C_{+}^{\mathcal{I}} \subseteq C_{-}^{\mathcal{I}} \cup D_{+}^{\mathcal{I}}$.

Corollary 4.2. Let $\mathcal{I}$ be a three-valued interpretation, $\langle\mathcal{R}, \mathcal{T}, \mathcal{A}\rangle$ be a knowledge base, $C$ be a concept, and a be an individual. Then:

- if $\mathcal{I}$ is an s-model of $\langle\mathcal{R}, \mathcal{T}, \mathcal{A}\rangle$ then it is also an m-model of $\langle\mathcal{R}, \mathcal{T}, \mathcal{A}\rangle$

- if $\mathcal{I}$ is an $\mathrm{m}$-model of $\langle\mathcal{R}, \mathcal{T}, \mathcal{A}\rangle$ then it is also a w-model of $\langle\mathcal{R}, \mathcal{T}, \mathcal{A}\rangle$

- if $\langle\mathcal{R}, \mathcal{T}, \mathcal{A}\rangle$ is s-satisfiable then it is also $\mathrm{m}$-satisfiable

- if $\langle\mathcal{R}, \mathcal{T}, \mathcal{A}\rangle$ is $\mathrm{m}$-satisfiable then it is also $\mathrm{w}$-satisfiable

- if $\langle\mathcal{R}, \mathcal{T}, \mathcal{A}\rangle \models{ }_{3}^{\mathrm{w}} C(a)$ then $\langle\mathcal{R}, \mathcal{T}, \mathcal{A}\rangle \models_{3}^{\mathrm{m}} C(a)$

- if $\langle\mathcal{R}, \mathcal{T}, \mathcal{A}\rangle \models_{3}^{\mathrm{m}} C(a)$ then $\langle\mathcal{R}, \mathcal{T}, \mathcal{A}\rangle \models_{3}^{\mathrm{s}} C(a)$.

Every two-valued interpretation $\mathcal{I}$ can be treated as a three-valued interpretation $\mathcal{I}^{\prime}$ with $\Delta^{\mathcal{I}^{\prime}}=\Delta^{\mathcal{I}}, A_{+}^{\mathcal{I}^{\prime}}=A^{\mathcal{I}}$ and $A_{-}^{\mathcal{I}^{\prime}}=\Delta^{\mathcal{I}} \backslash A^{\mathcal{I}}$ for every concept name $A, R^{\mathcal{I}^{\prime}}=R^{\mathcal{I}}$ for every role name $R$, and $a^{\mathcal{I}^{\prime}}=a^{\mathcal{I}}$ for every individual name $a$.

Clearly, if $\mathcal{I}$ is a two-valued interpretation then $\mathcal{I} \models C(a)$ iff $\mathcal{I}={ }_{3} C(a)$.

The proof of the following proposition is straightforward, using Corollary 4.2

Proposition 4.3. Let $\mathcal{I}$ be a two-valued interpretation, $\langle\mathcal{R}, \mathcal{T}, \mathcal{A}\rangle$ be a knowledge base, $C$ be a concept, and a be an individual. Then:

- if $\mathcal{I} \models\langle\mathcal{R}, \mathcal{T}, \mathcal{A}\rangle$ then $\mathcal{I} \models{ }_{3}^{\mathrm{s}}\langle\mathcal{R}, \mathcal{T}, \mathcal{A}\rangle$

- if $\langle\mathcal{R}, \mathcal{T}, \mathcal{A}\rangle \models{ }_{3}^{t} C(a)$, where $t \in\{\mathrm{s}, \mathrm{m}, \mathrm{w}\}$, then $\langle\mathcal{R}, \mathcal{T}, \mathcal{A}\rangle \models C(a)$.

\footnotetext{
${ }^{2}$ In addition to negative evidence resulting from the fact that $\neg L R\left(s_{1}\right)$.
} 
The intuitive meaning of $T$ is "always true" and of $\perp$ is "always false". For this reason, no admissible semantics would accept $T \sqsubseteq \perp$ as a terminological axiom. That is, there exist always unsatisfiable knowledge bases if one allows terminological axioms of the form $T \sqsubseteq \perp$. A natural question is when a knowledge base is $t$-satisfiable, for $t \in\{\mathrm{w}, \mathrm{m}, \mathrm{s}\}$. We give below a syntactic sufficient condition for $t$-satisfiability.

We first define $\top$-safety and $\perp$-safety. The intuition is that $C$ is $\top$-safe (resp. $\perp$-safe) if it is "free" of effects of $T_{-}^{\mathcal{I}}=\emptyset$ (resp. $\left.\perp_{+}^{\mathcal{I}}=\emptyset\right)$ and we can choose an interpretation $\mathcal{I}$ such that $C_{-}^{\mathcal{I}}=\Delta^{\mathcal{I}}$ (resp. $C_{+}^{\mathcal{I}}=\Delta^{\mathcal{I}}$ ). The set of $\top$-safe (resp. $\perp$-safe) concepts is the smallest set of concepts such that:

- $\top$ is $\perp$-safe and $\perp$ is $T$-safe

- every atomic concept $A$ is $\top$-safe and $\perp$-safe

- if $C$ is $\top$-safe (resp. $\perp$-safe) then $\neg C$ is $\perp$-safe (resp. $\top$-safe)

- if $C$ or $D$ is $\top$-safe then $C \sqcap D$ is $\top$-safe

- if $C$ or $D$ is $\perp$-safe then $C \sqcup D$ is $\perp$-safe

- if both $C$ and $D$ are $\perp$-safe then $C \sqcap D$ is $\perp$-safe

- if both $C$ and $D$ are $\top$-safe then $C \sqcup D$ is $\top$-safe

- if $C$ is $\top$-safe (resp. $\perp$-safe) then $\forall R . C$ and $\exists R$. $C$ are $\top$-safe (resp. $\perp$-safe)

- if $C$ is $\perp$-safe then $\geq n R . C$ is $\perp$-safe and $\leq n R . C$ is $\top$-safe

- if $C$ is $\top$-safe then $\leq n$ R. $C$ is $\perp$-safe

- if $C$ is $\top$-safe and $n>0$ then $\geq n R . C$ is $\top$-safe.

A role axiom $C \sqsubseteq D$ is w-safe if $C$ is $\top$-safe or $D$ is $\perp$-safe. It is m-safe if $D$ is $\perp$-safe and it is s-safe if $C$ is $\top$-safe and $D$ is $\perp$-safe. A role axiom $C=D$ is $t$-safe, where $t \in\{\mathrm{w}, \mathrm{m}, \mathrm{s}\}$, if $C \sqsubseteq D$ and $D \sqsubseteq C$ are $t$-safe. A TBox is $t$-safe, where $t \in\{\mathrm{w}, \mathrm{m}, \mathrm{s}\}$, if all of its axioms are $t$-safe.

A concept assertion $C(a)$ is safe if $C$ is $\perp$-safe. An ABox is safe if all of its concept assertions are safe. A knowledge base $\langle\mathcal{R}, \mathcal{T}, \mathcal{A}\rangle$ is $t$-safe, where $t \in\{\mathrm{w}, \mathrm{m}, \mathrm{s}\}$, if $\mathcal{T}$ is $t$-safe and $\mathcal{A}$ is safe.

A concept is in negation normal form (NNF) if it uses $\neg$ only immediately before concept names. Using De Morgan's laws with the special cases

$$
\begin{aligned}
& \neg(\leq n \text { R.C })=\geq(n+1) \text { R.C } \quad \neg(\geq(n+1) R . C)=\leq n \text { R.C } \\
& \neg(\geq 0 \text { R.C })=\perp,
\end{aligned}
$$

every concept $C$ can be translated into a concept $C^{\prime}$ in NNF which is equivalent to $C$ w.r.t. both two-valued and three-valued semantics in the sense that $C^{\mathcal{I}}=C^{{ }^{\mathcal{I}}}$ for every two-valued or three-valued interpretation $\mathcal{I}$. A knowledge base is in NNF if it uses only concepts in NNF. We have the following straightforward proposition.

\section{Proposition 4.4}

- If a knowledge base is $\mathrm{s}$-safe then it is also m-safe.

- If a knowledge base is $\mathrm{m}$-safe then it is also $\mathrm{w}$-safe.

- If a knowledge base does not use $\top, \perp$ and concepts of the form $\geq 0 R$.C then it is $t$-safe, for any $t \in\{\mathrm{w}, \mathrm{m}, \mathrm{s}\}$.

- If a knowledge base is in NNF and does not use $\perp$ and concepts of the form $\leq n$ R.C then it is m-safe. 
Theorem 4.5. If a knowledge base $\langle\mathcal{R}, \mathcal{T}, \mathcal{A}\rangle$ is $t$-safe, where $t \in\{\mathrm{w}, \mathrm{m}, \mathrm{s}\}$, then it is $t$-satisfiable.

Proof. Let $n$ be the largest number occurring in the knowledge base, or 1 if no number restrictions are used for the knowledge base. Take any three-valued interpretation $\mathcal{I}$ such that $\left|\Delta^{\mathcal{I}}\right|=n+1, A_{+}^{\mathcal{I}}=A_{-}^{\mathcal{I}}=\Delta^{\mathcal{I}}$ for any concept name $A$, and $R^{\mathcal{I}}=\Delta^{\mathcal{I}} \times \Delta^{\mathcal{I}}$ for any role name $R$. It can be proved by induction on the structure of $C$ that:

- if $C$ occurs in the knowledge base and is $T$-safe then $C_{-}^{\mathcal{I}}=\Delta^{\mathcal{I}}$

- if $C$ occurs in the knowledge base and is $\perp$-safe then $C_{+}^{\overline{\mathcal{I}}}=\Delta^{\mathcal{I}}$.

Using this it is easy to see that if $\langle\mathcal{R}, \mathcal{T}, \mathcal{A}\rangle$ is $t$-safe, where $t \in\{\mathrm{w}, \mathrm{m}, \mathrm{s}\}$, then $\mathcal{I}$ is a $t$-model of $\langle\mathcal{R}, \mathcal{T}, \mathcal{A}\rangle$.

\section{The Relationship with Four-Valued Semantics}

Four-valued interpretations are defined similarly as three-valued interpretations but without the condition that $A_{+}^{\mathcal{I}} \cup A_{-}^{\mathcal{I}}=\Delta^{\mathcal{I}}$ for every concept name $A$. The papers [10]11] use non-traditional inclusion axioms $C \mapsto D$ ("material inclusion"), $C \sqsubset D$ ("internal inclusion") and $C \rightarrow D$ ("strong inclusion"). The inclusion $C \mapsto D$ (resp. $C \sqsubset D, C \rightarrow D$ ) corresponds to our inclusion $C \sqsubseteq D$ w.r.t. semantics w (resp. m, s). The relationship between our three-valued semantics and the four-valued semantics $\models_{4}$ given in [10]11] can be characterized as follows. Having a (traditional) knowledge base $\langle\mathcal{R}, \mathcal{T}, \mathcal{A}\rangle$, when $\sqsubseteq$ is interpreted as:

- the material inclusion, then $\langle\mathcal{R}, \mathcal{T}, \mathcal{A}\rangle \models_{4} C(a)$ implies $\langle\mathcal{R}, \mathcal{T}, \mathcal{A}\rangle \models_{3}^{\mathrm{w}} C(a)$

- the internal inclusion, then $\langle\mathcal{R}, \mathcal{T}, \mathcal{A}\rangle \models_{4} C(a)$ implies $\langle\mathcal{R}, \mathcal{T}, \mathcal{A}\rangle \models_{3}^{\mathrm{m}} C(a)$

- the strong inclusion, then $\langle\mathcal{R}, \mathcal{T}, \mathcal{A}\rangle \models_{4} C(a)$ implies $\langle\mathcal{R}, \mathcal{T}, \mathcal{A}\rangle \models_{3}^{\mathrm{s}} C(a)$.

Therefore, fixing the meaning of $\sqsubseteq$ and using our appropriate three-valued semantics $t \in\{\mathrm{w}, \mathrm{m}, \mathrm{s}\}$, by Proposition 4.3 , the problem of checking $\langle\mathcal{R}, \mathcal{T}, \mathcal{A}\rangle \models_{3}^{t} C(a)$ better approximates the traditional instance checking problem $\langle\mathcal{R}, \mathcal{T}, \mathcal{A}\rangle \models C(a)$ than the problem of checking $\langle\mathcal{R}, \mathcal{T}, \mathcal{A}\rangle \models_{4} C(a)$ does. Thus, for the instance checking problem w.r.t. a $t$-safe knowledge base, our three-valued semantics $t$ is better than the four-valued semantics defined in [10[11]. Note that the classes of $t$-safe knowledge bases, for $t \in\{\mathrm{w}, \mathrm{m}, \mathrm{s}\}$, are very large, and it is rather not possible to formulate a more general syntactic condition of safeness.

The following example shows that the approaches based on Belnap's logic, like [10]11], discussed above, sometimes lead to counter-intuitive results.

Example 5.1 (Example 3.2 continued). Recall that we have considered a web agent looking for low risk stocks, promising big gain. Assume that for a given stock $s$ a semantic web service has inconsistent information whether $s$ is promising big gain and has no information whether $s$ is of low risk. Consider the following two agent's queries: 
1. $(L R \sqcap B G)(s)$, expressing the fact that $s$ is of low risk and promising big gain: the semantics of [10]11], results in $\mathfrak{f}$, while in our semantics is $\mathbf{i}^{\text {? }}$.

2. $(L R \sqcup B G)(s)$, expressing the fact that $s$ is of low risk or promising big gain: the semantics of [10[11], results in $\mathfrak{t}$, while in our semantics it again results in $\mathfrak{i}$.

Both in the case of conjunction and of disjunction the results under our semantics are much more intuitive and informative than in the case of Belnap-like semantics.

In [17], Zhang et al define weak and strong quasi-classical semantics for $\mathcal{S H} \mathcal{H} \mathcal{Q}$, which are four-valued semantics and will be denoted here by $\models_{4}^{w}$ and $\models_{4}^{s}$. For the instance checking problem, the weak quasi-classical semantics is weaker than our weak threevalued semantics in the sense that if $\langle\mathcal{R}, \mathcal{T}, \mathcal{A}\rangle \models_{4}^{w} C(a)$ then $\langle\mathcal{R}, \mathcal{T}, \mathcal{A}\rangle \models{ }_{3}^{\mathrm{w}} C(a)$. Comparing our semantics $\models_{3}^{\text {s }}$ (i.e. s) with $\models_{4}^{s}$, neither of them is stronger than the other. The relationship between these semantics can be characterized as follows. First, axioms of the form $C \sqsubseteq C$ is not valid w.r.t. the semantics $\models_{4}^{s}$, which is quite unusual. Second, extending $\models_{4}^{s}$ with axioms $A \sqsubseteq A$ for all atomic concepts $A$ results in a three-valued semantics that differs from our semantics $\models_{3}^{\mathrm{s}}$ in that it assumes $\mathfrak{t} \wedge \mathfrak{i}=\mathfrak{t}$ and $\mathfrak{f} \vee \mathfrak{i}=\mathfrak{f}$, while our semantics $\|_{3}^{\mathrm{s}}$ assumes $\mathfrak{t} \wedge \boldsymbol{i}=\boldsymbol{i}$ and $\mathfrak{f} \vee \boldsymbol{i}=\mathbf{i}$. Moreover, the same problem as indicated in Example 5.1, applies to both weak and strong semantics proposed in [17], so the assumptions of our semantics are more appropriate.

\section{A Translation into Two-Valued Description Logic}

For $A \in \mathrm{CNAMES}$, let $A_{+}$and $A_{-}$be new concept names. Let CNAMES ${ }^{\prime}=\left\{A_{+}, A_{-} \mid\right.$ $A \in \mathrm{CNAmES}\}$. For a concept $C$ in the language using CNAMES, let $\pi_{+}(C)$ and $\pi_{-}(C)$ be the concepts defined as follows:

$$
\begin{array}{rlrl}
\pi_{+}(\top) & =\top & \pi_{-}(\top) & =\perp \\
\pi_{+}(\perp) & =\perp & \pi_{-}(\perp) & =\top \\
\pi_{+}(A) & =A_{+} \\
\pi_{-}(A) & =A_{-} \\
\pi_{+}(\neg C) & =\pi_{-}(C) & \pi_{-}(\neg C) & =\pi_{+}(C) \\
\pi_{+}(C \sqcap D) & =\pi_{+}(C) \sqcap \pi_{+}(D) & \pi_{-}(C \sqcap D) & =\pi_{-}(C) \sqcup \pi_{-}(D) \\
\pi_{+}(C \sqcup D) & =\pi_{+}(C) \sqcup \pi_{+}(D) & \pi_{-}(C \sqcup D) & =\pi_{-}(C) \sqcap \pi_{-}(D) \\
\pi_{+}(\forall R . C) & =\forall R . \pi_{+}(C) & \pi_{-}(\forall R . C) & =\exists R . \pi_{-}(C) \\
\pi_{+}(\exists R . C) & =\exists R . \pi_{+}(C) & \pi_{-}(\exists R . C) & =\forall R . \pi_{-}(C) \\
\pi_{+}(\geq n R . C) & =\geq n R . \pi_{+}(C) & \pi_{-}(\geq(n+1) R . C) & =\leq n R . \neg \pi_{-}(C) \\
\pi_{+}(\leq n R . C) & =\leq n R . \neg \pi_{-}(C) & \pi_{-}(\geq 0 R . C) & =\perp \\
\pi_{-}(\leq n R . C) & =\geq(n+1) R . \pi_{+}(C)
\end{array}
$$

For concepts $C$ and $D$ in the language using CNAMES and for $t \in\{\mathrm{w}, \mathrm{m}, \mathrm{s}\}$, define $\pi_{t}(C \sqsubseteq D)$ and $\pi_{t}(C=D)$ as follows:

$$
\begin{aligned}
\pi_{\mathrm{w}}(C \sqsubseteq D) & =\left\{\top \sqsubseteq \pi_{-}(C) \sqcup \pi_{+}(D)\right\} \\
\pi_{\mathrm{m}}(C \sqsubseteq D) & =\left\{\pi_{+}(C) \sqsubseteq \pi_{+}(D)\right\} \\
\pi_{\mathrm{s}}(C \sqsubseteq D) & =\left\{\pi_{+}(C) \sqsubseteq \pi_{+}(D), \pi_{-}(D) \sqsubseteq \pi_{-}(C)\right\} \\
\pi_{t}(C=D) & =\pi_{t}(C \sqsubseteq D) \cup \pi_{t}(D \sqsubseteq C)
\end{aligned}
$$

\footnotetext{
${ }^{3}$ Since we identify $\mathfrak{u}$ with $\mathfrak{i}$.
} 
For a knowledge base $\langle\mathcal{R}, \mathcal{T}, \mathcal{A}\rangle$ in the language using CNAMES and for $t \in\{\mathrm{w}, \mathrm{m}, \mathrm{s}\}$, define $\pi_{t}(\langle\mathcal{R}, \mathcal{T}, \mathcal{A}\rangle)$ to be the knowledge base $\left\langle\mathcal{R}, \pi_{t}(\mathcal{T}), \pi(\mathcal{A})\right\rangle$ (in the language using CNAMES'), where:

$$
\begin{aligned}
\pi_{t}(\mathcal{T}) & =\bigcup_{\varphi \in \mathcal{T}} \pi_{t}(\varphi) \cup\left\{\top \sqsubseteq A_{+} \sqcup A_{-} \mid A \in \mathrm{CNAMES}\right\} \\
\pi(\mathcal{A}) & =\left\{\pi_{+}(C)(a) \mid C(a) \in \mathcal{A}\right\} \cup\{R(a, b) \mid R(a, b) \in \mathcal{A}\}
\end{aligned}
$$

Note that the size of $\pi_{t}(\langle\mathcal{R}, \mathcal{T}, \mathcal{A}\rangle)$ is linear in the size of $\langle\mathcal{R}, \mathcal{T}, \mathcal{A}\rangle$ and CNAMES.

Theorem 6.1. Let $\langle\mathcal{R}, \mathcal{T}, \mathcal{A}\rangle$ be a knowledge base and $C$ be a concept in the language using CNAMES. If $a$ is an individual and $t \in\{\mathrm{w}, \mathrm{m}, \mathrm{s}\}$ then $\langle\mathcal{R}, \mathcal{T}, \mathcal{A}\rangle \models_{3}^{t} C(a)$ iff $\pi_{t}(\langle\mathcal{R}, \mathcal{T}, \mathcal{A}\rangle) \models \pi_{+}(C)(a)$.

Proof. Consider the right to left implication and assume that $\pi_{t}(\langle\mathcal{R}, \mathcal{T}, \mathcal{A}\rangle) \models$ $\pi_{+}(C)(a)$. Let $\mathcal{I}$ be a $t$-model of $\langle\mathcal{R}, \mathcal{T}, \mathcal{A}\rangle$ in the language using CNAMES. We show that $\mathcal{I} \models_{3} C(a)$. Let $\mathcal{I}^{\prime}$ be the two-valued interpretation in the language using CNAMES' specified as follows:

$-\Delta^{\mathcal{I}^{\prime}}=\Delta^{\mathcal{I}}$

- $\left(A_{+}\right)^{\mathcal{I}^{\prime}}=A_{+}^{\mathcal{I}}$ and $\left(A_{-}\right)^{\mathcal{I}^{\prime}}=A_{-}^{\mathcal{I}}$ for every concept name $A \in$ CNAMES

$-R^{\mathcal{I}^{\prime}}=R^{\mathcal{I}}$ for every role name

- $a^{\mathcal{I}^{\prime}}=a^{\mathcal{I}}$ for every individual name $a$.

Let $D$ be a concept in the language using CNAMES. It is easy to prove by induction on the structure of $D$ that $D^{\mathcal{I}}=\left\langle\left(\pi_{+}(D)\right)^{\mathcal{I}^{\prime}},\left(\pi_{-}(D)\right)^{\mathcal{I}^{\prime}}\right\rangle$. Using this and the fact that $\mathcal{I}$ is a $t$-model of $\langle\mathcal{R}, \mathcal{T}, \mathcal{A}\rangle$ we derive that $\mathcal{I}^{\prime}$ is a model of $\pi_{t}(\langle\mathcal{R}, \mathcal{T}, \mathcal{A}\rangle)$. Hence $\mathcal{I}^{\prime} \models \pi_{+}(C)(a)$. It follows that $\mathcal{I} \models_{3} C(a)$.

The left to right implication can be proved analogously.

To check whether $\pi_{t}(\langle\mathcal{R}, \mathcal{T}, \mathcal{A}\rangle) \models \pi_{+}(C)(a)$ one can use, e.g., the tableau method given in [8]. We have the following corollary of Theorem 6.1 by taking $C=\perp$.

Corollary 6.2. Let $\langle\mathcal{R}, \mathcal{T}, \mathcal{A}\rangle$ be a knowledge base in the language using CNAMES and let $t \in\{\mathrm{w}, \mathrm{m}, \mathrm{s}\}$. Then $\langle\mathcal{R}, \mathcal{T}, \mathcal{A}\rangle$ is t-satisfiable iff $\pi_{t}(\langle\mathcal{R}, \mathcal{T}, \mathcal{A}\rangle)$ is satisfiable (w.r.t. two-valued semantics).

\section{References}

1. Baader, F., Calvanese, D., McGuinness, D.L., Nardi, D., Patel-Schneider, P.F. (eds.): Description Logic Handbook. Cambridge University Press, Cambridge (2002)

2. Belnap, N.D.: How a computer should think. In: Ryle, G. (ed.) Contemporary Aspects of Philosophy, Stocksfield, pp. 30-55. Oriel Press (1977)

3. Belnap, N.D.: A useful four-valued logic. In: Eptein, G., Dunn, J.M. (eds.) Modern Uses of Many Valued Logic, pp. 8-37. Reidel, Dordrechtz (1977)

4. Béziau, J.-Y., Carnielli, W., Gabbay, D.M. (eds.): Handbook of Paraconsistency. Logic and cognitive systems, vol. 9. College Publications (2007)

5. Bloesch, A.: A tableau style proof system for two paraconsistent logics. Notre Dame Journal of Formal Logic 34(2), 295-301 (1993) 
6. Doherty, P., Łukaszewicz, W., Skowron, A., Szałas, A.: Knowledge Representation Techniques. In: A Rough Set Approach, Springer, Heidelberg (2006)

7. Horrocks, I., Patel-Schneider, P.F., van Harmelen, F.: From SHIQ and RDF to OWL: The making of a web ontology language. Journal of Web Semantics 1(1), 7-26 (2003)

8. Horrocks, I., Sattler, U., Tobies, S.: Reasoning with individuals for the description logic SHIQ. In: McAllester, D. (ed.) CADE 2000. LNCS, vol. 1831, pp. 482-496. Springer, Heidelberg (2000)

9. Kleene, S.C.: Introduction to Metamathematics. D. Van Nostrand, Princeton (1952)

10. Ma, Y., Hitzler, P.: Paraconsistent reasoning for OWL 2. In: Polleres, A. (ed.) RR 2009. LNCS, vol. 5837, pp. 197-211. Springer, Heidelberg (2009)

11. Ma, Y., Hitzler, P., Lin, Z.: Paraconsistent reasoning for expressive and tractable description logics. In: Proc. of Description Logics (2008)

12. Maluszyński, J., Szałas, A., Vitória, A.: Paraconsistent logic programs with four-valued rough sets. In: Chan, C.-C., Grzymala-Busse, J.W., Ziarko, W.P. (eds.) RSCTC 2008. LNCS (LNAI), vol. 5306, pp. 41-51. Springer, Heidelberg (2008)

13. Meghini, C., Straccia, U.: A relevance terminological logic for information retrieval. In: Proc. of SIGIR 1996, pp. 197-205. ACM, New York (1996)

14. Odintsov, S.P., Wansing, H.: Inconsistency-tolerant description logic. part II: A tableau algorithm for CACL ${ }^{\mathrm{C}}$. Journal of Applied Logic 6(3), 343-360 (2008)

15. Straccia, U.: A sequent calculus for reasoning in four-valued description logics. In: Galmiche, D. (ed.) TABLEAUX 1997. LNCS, vol. 1227, pp. 343-357. Springer, Heidelberg (1997)

16. Vitória, A., Maluszyński, J., Szałas, A.: Modeling and reasoning in paraconsistent rough sets. Fundamenta Informaticae 97(4), 405-438 (2009)

17. Zhang, X., Qi, G., Ma, Y., Lin, Z.: Quasi-classical semantics for expressive description logics. In: Proc. of Description Logics (2009) 